\title{
Spectrographic and imaging observations of solar type $U$ radio bursts
}

\author{
H. Aurass ${ }^{1}$ and K.-L. Klein ${ }^{2}$ \\ 1 Astrophysikalisches Institut Potsdam, Observatorium für solare Radioastronomie, Telegrafenberg A31, D-14473 Potsdam, \\ Germany \\ e-mail: haurass@aip.de \\ 2 DASOP, CNRS-URA 2080, Observatoire de Paris, Section de Meudon, F-92195 Meudon, France \\ e-mail: klein@obspm.fr
}

Received March 4; accepted September 18, 1996

\begin{abstract}
A sample of 23 type $\mathrm{U}$ bursts is studied by use of spectrographic and imaging observations at decimetric and metric wavelengths (spectrograph of Potsdam - Tremsdorf Solar Radio Observatory, Nançay multifrequency radioheliograph of Paris Observatory). The radio source locations are compared with Yohkoh soft X-ray or Meudon $\mathrm{H}_{\alpha}$ images. The main results are:
\end{abstract}

1. The sample is consistent with isotropic emission from U bursts uniformly distributed over the disk.

2. The configuration corresponds to bursts being emitted by electron beams in loop structures: the rising and the descending branches have spatially separated sources. Under appropriate observing conditions they can be shown to project to different sides of the neutral line of the photospheric magnetic field. Underlying plasma loop structures with the same topology could in several cases be identified in Yohkoh SXT images.

3. On many occasions complex source patterns are observed. Type III bursts and ascending type U branches that occur together within $\leq 1 \mathrm{~s}$ give evidence for cotemporal beam injection into structures of different connectivity. Repetitive brightenings near the site of the ascending $U$ branch require repeated beam injection or the formation of counterstreaming electron beams in coronal loops.

4. A majority of the events has higher starting frequency, higher flux density and a smaller source in the ascending branch than in the descending branch, but examples of the opposite situation are also found. The degree of circular polarization is generally low and difficult to measure. No systematic behaviour is recognized.

5. Simultaneous type U bursts were found in one case with a frequency ratio near 1.7. The location of the

Send offprint requests to: H. Aurass sources during the two lanes excludes their interpretation as a fundamental - harmonic pair.

6. Type U bursts are found in transient large-scale structures as well as in configurations that are stable over several hours. The repetitive occurrence of type U bursts with a homologous source pattern (in space and time) has been detected during up to 4 hours. Such stable conditions of the beam injection and the propagation path may be in conflict with stochastic or chaotic beam injection dynamics.

Key words: Sun: activity - Sun: corona - Sun: flares - Sun: magnetic field — Sun: particle emission Sun: radio radiation

\section{Introduction}

Fast drift bursts are among the best understood features of the nonthermal decimeter/meterwave emission of the solar corona. The most frequent type III bursts are induced by beams of mildly relativistic electrons accelerated in an active region and travelling along open magnetic field lines toward regions of lower densities of the background corona (cf. reviews by Goldman \& Smith 1985; Suzuki \& Dulk 1985; Pick \& van den Oord 1990). The negatively drifting signature in the spectrogram is due to the transformation of plasma waves into electromagnetic waves near the local plasma frequency (fundamental mode, F) and/or twice this value (first harmonic mode, $\mathrm{H}$ ) possibly leading to simultaneous emission at harmonically related frequencies. Positively drifting type III-like bursts (drifting to higher frequencies, "reverse drift bursts") are attributed to electron beams propagating toward regions of higher densities.

Type U bursts consist of a combination of a type III burst and a reverse drift burst with a continuous 
turning region in the spectrum thus leading to an "inverted U" spectral signature (Maxwell \& Swarup 1958) reported from decimeter to dekameter waves (Stone \& Fainberg 1971; Aschwanden et al. 1992). The incomplete species with missing descending spectral branch but an emission "flag" after a turning point is called J bursts. When a characteristic type III-like burst follows the type $\mathrm{U}$ signature the whole burst is referred to as type $\mathrm{N}$ or $\mathrm{U}(\mathrm{N})$ burst (Caroubalos et al. 1987). If continuum emission follows a type III or $U$ burst it is called a type $\mathrm{V}$ burst. Occasionally fundamental - harmonic $\mathrm{U}$ and J burst patterns (Haddock \& Takakura 1965; Labrum \& Stewart 1970) have been reported.

Type U bursts are much rarer than type III bursts. The reason is presently unknown, especially since closed field lines inferred from plasma tubes are a usual and typical morphological feature of the solar corona (e.g. Priest 1978; Bray et al. 1991). Early work on type U bursts (Fokker 1970; Labrum \& Stewart 1970; Sheridan et al. 1973; Suzuki 1978) showed that the sources of the ascending and descending branch at meter wavelengths trace large scale loop structures which were seen in a few cases to overlie soft X-ray loops observed by Skylab (Stewart \& Vorpahl 1977). In case studies, Aurass et al. (1994) and Pick et al. (1994) were able to separate the ascending and descending branch and to localize them on either side of soft X-ray loops seen by the Yohkoh SXT. Aschwanden et al. (1992) observed decimeter type U sources around the summit of extrapolated magnetic field lines. While these results suggest that type U bursts are due to electron beams guided along closed magnetic field structures in the corona, the expected difference between the sense of circular polarization of the ascending and descending U burst branch was only occasionally observed (Sheridan et al. 1973; Benz et al. 1977, 1979; Suzuki 1978).

A comprehensive study of type U burst observations including spectral and spatial resolution as well as polarization data with high time resolution and the comparison with images of coronal structures therefore seems appropriate. We present a sample of events in the attempt to give a full account of the status of available observations and their relation to simple models of beam propagation in the corona. It is also our aim to point out some of the difficulties with the identification of "true" type U signatures in spectral records. The data are presented as a catalogue given in the Appendix together with a brief description of the instrumentation. Section 2 summarizes the main content of the catalogue.

\section{Catalogue summary and brief discussion of the observations}

The studied sample consists of 23 events. Gross spectral and spatial characteristics are summarized in Tables 1 to 3 , and the individual events are described in the Appendix.

\subsection{Global characteristics of the dynamic spectra}

The bursts extend from decimetric to metric waves, with turnover frequencies ranging from 100 to $380 \mathrm{MHz}$ and starting frequencies from 170 to $460 \mathrm{MHz}$ (Table 1). The average total bandwidth is about half the centre frequency (Table 2). These are not statistical properties of type U bursts observed by the Tremsdorf spectrograph, but are largely due to the requirement that the bursts should be observable by the Nançay radioheliograph (NRH). Two cases have the spectrum and the spatial configuration of type $\mathrm{U}(\mathrm{N})$ bursts.

\subsection{Spatial distribution of type $U$ burst sources}

The vast majority of the bursts is located near or above the limb in projection onto the plane of the sky. This is at least partly due to foreshortening and to the possibility to observe behind-limb events. If isotropically emitting radio sources are placed uniformly in longitude at an altitude of e.g. $R_{\odot} / 3$ above the photosphere, $63 \%$ of the observed sources will appear projected above the limb. This is consistent with the distribution of the ascending branch source A of our type U sample: $38 \%$ are at projected distances below $1 R_{\odot}, 62 \%$ at distances between 1 and $1.4 R_{\odot}$ (multiple bursts from the same day and the same site have been counted as a single event). The distribution of our small sample is therefore consistent with the assumption of isotropic emission from uniformly distributed type U burst sources on the Sun, in agreement with earlier conclusions based on the location of $\mathrm{H}_{\alpha}$ flares associated with such radio bursts (Fokker 1970).

\subsection{Association with flares}

The last two columns of Table 1 list the $\mathrm{H}_{\alpha}$ and soft X-ray emissions which accompany the $\mathrm{U}$ bursts. It is clear from a comparison with Table 3 that even when the type U burst is associated with active regions on the disk it does not necessarily occur with an $\mathrm{H}_{\alpha}$ flare (SGD Comprehensive Reports; e.g. 4 Nov. and 25 Jan. 1994, 13 Nov. 1992).

Hard X-ray observations are not available during most of the events of interest. As a proxy (cf. Dennis \& Zarro 1993), the time intervals where the derivative of the soft $\mathrm{X}$-ray flux is positive are given in the last column of Table 1 (GOES satellites; data kindly provided by SDAC at Goddard Space Flight Center). The majority of the U/III burst groups (14/23) occurs together with weak X-ray brightenings, but a significant number (9/23) has no or probably no counterpart detected by GOES. Three of them occur on the disk. The 13 Nov. 1992 burst shows that this is at least in part a problem of sensitivity, since Yohkoh-SXT detects a jet in time coincidence with the U-burst (Aurass et al. 1994). Our selection of simple and spectrally well-defined events likely favours the tendency of weak flare association. 
Table 1. The U burst sample

\begin{tabular}{|c|c|c|c|c|c|c|c|c|c|}
\hline Date & Start & Top & End & Type & $\mathrm{Up}$ & Down & Loop & $\mathrm{H}_{\alpha}$ & SXR \\
\hline $\begin{array}{r}04 \text { Nov. } \\
94\end{array}$ & $\begin{array}{c}1139: 17 \\
250\end{array}$ & $\begin{array}{c}1139: 20.5 \\
127\end{array}$ & $\begin{array}{l}1139: 23 \\
170-200\end{array}$ & III-U & 1 & 2 & ic & no & (no) \\
\hline $\begin{array}{r}25 \text { Oct. } \\
94 \\
25 \text { Oct. } \\
94 \\
25 \text { Oct. } \\
94 \\
25 \text { Oct. } \\
94 \\
\end{array}$ & $\begin{array}{c}1007: 47.0 \\
460 \\
1144: 13 \\
220 \\
1231: 23 \\
290 \\
1331: 45 \\
240 \\
\end{array}$ & $\begin{array}{c}1007: 47.6 \\
380 \\
1144: 16 \\
140 \\
1231: 25 \\
130 \\
(13: 31: 47) \\
(140) \\
\end{array}$ & $\begin{array}{c}1007: 48.0 \\
500 \\
1144: 18.5 \\
230 \\
1231: 28.5 \\
340 \\
1331: 50 \\
350 \\
\end{array}$ & $\begin{array}{l}\text { III-U } \\
\text { III-U } \\
\text { III-U }\end{array}$ & $\begin{array}{c}1 \\
2 \\
(1)\end{array}$ & 1 & $\begin{array}{l}\mathrm{ar} \\
\mathrm{aq} \\
\mathrm{aq} \\
\mathrm{aq}\end{array}$ & $\begin{array}{c}\text { 1N S08 W12 } \\
0940-1236 \\
\text { no } \\
<1236>1421 \\
\end{array}$ & $\begin{array}{c}\mathrm{C} 4.70959 \\
0940-1009 \\
(<\mathrm{C} 1.11144) \\
(1144-1145) \\
<\mathrm{B} 7.11232 \\
1231-1232 \\
<\mathrm{B} 4.21331 \\
1331-1332 \\
\end{array}$ \\
\hline $\begin{array}{r}28 \text { Jan. } \\
94 \\
28 \text { Jan. } \\
94 \\
28 \text { Jan. } \\
94 \mathrm{~F} \\
\mathrm{H}\end{array}$ & $\begin{array}{c}1123: 55 \\
290 \\
1124: 56.5 \\
350 \\
1129: 09 \\
170-200 \\
280\end{array}$ & $\begin{array}{c}1123: 57 \\
220 \\
1124: 58.5 \\
255 \\
1129: 11 \\
125 \\
215 \\
\end{array}$ & $\begin{array}{c}1124: 00 \\
250 \\
1125: 00.5 \\
290 \\
1129: 21 \\
300 \\
250\end{array}$ & $\begin{array}{l}\text { III-U } \\
\text { III-U } \\
\text { III-U }\end{array}$ & $\begin{array}{l}1 \\
1\end{array}$ & $\begin{array}{l}1 \\
-\end{array}$ & $\begin{array}{l}\mathrm{aq} \\
\mathrm{aq} \\
\mathrm{aq}\end{array}$ & $\begin{array}{c}\text { no } \\
>1513\end{array}$ & $\begin{array}{l}\text { C1.2 } 1126 \\
1123-1128\end{array}$ \\
\hline $\begin{array}{r}25 \text { Jan. } \\
94 \\
\end{array}$ & $\begin{array}{c}1052: 54 \\
280 \\
\end{array}$ & $\begin{array}{c}1053: 00 \\
130 \\
\end{array}$ & $\begin{array}{l}1054: 04 \\
170-200 \\
\end{array}$ & U-III & 1 & 2 & (ic) & $\begin{array}{c}\text { no } \\
<1000>1246 \\
\end{array}$ & $\begin{array}{c}<\mathrm{B} 6.81051 \\
1050-1053 \\
\end{array}$ \\
\hline $\begin{array}{r}03 \text { Jun. } \\
93 \\
\end{array}$ & $\begin{array}{l}1205: 36 \\
170-200 \\
\end{array}$ & $\begin{array}{c}1205: 39-42 \\
130 \\
\end{array}$ & $\begin{array}{l}1205: 48 \\
170-200 \\
\end{array}$ & $\begin{array}{l}\text { U with } \\
\text { gap }\end{array}$ & 1 & 1 & - & $\begin{array}{c}\text { no } \\
<1100>1326 \\
\end{array}$ & no \\
\hline $\begin{array}{r}14 \text { May } \\
93 \\
\end{array}$ & $\begin{array}{c}0921: 42 \\
440 \\
\end{array}$ & $\begin{array}{c}0921: 43 \\
310 \\
\end{array}$ & $\begin{array}{c}0921: 45 \\
400 \\
\end{array}$ & $\mathrm{U}$ & 1 & 2 & ic & $\begin{array}{c}\text { no } \\
<0546>1545\end{array}$ & no \\
\hline $\begin{array}{r}23 \text { Feb. } \\
93 \\
23 \text { Feb. } \\
93 \\
\end{array}$ & $\begin{array}{c}1134: 23 \\
400 \\
1138: 03 \\
400\end{array}$ & $\begin{array}{l}1134: 26 \\
170-230 \\
1138: 06 \\
170-210\end{array}$ & $\begin{array}{c}1134: 30 \\
320 \\
1138: 08 \\
350\end{array}$ & $\begin{array}{l}\text { III-U-III } \\
\text { (U)-III- } \\
\text { U-N }\end{array}$ & 1 & $\begin{array}{l}2 \\
2\end{array}$ & $\begin{array}{l}\text { ic } \\
\text { ic }\end{array}$ & $\begin{array}{c}\text { no } \\
\text { but SF S16 E20 } \\
1117-1128\end{array}$ & $\begin{array}{c}<\mathrm{B} 7.01134 \\
1133-1135 \\
\text { no }\end{array}$ \\
\hline $\begin{array}{r}15 \text { Jan. } \\
93 \\
\end{array}$ & $\begin{array}{c}0925: 17 \\
(250) \\
\end{array}$ & $\begin{array}{l}0925: 22 \\
100-120 \\
\end{array}$ & $\begin{array}{c}0925: 23 \\
(250) \\
\end{array}$ & $\begin{array}{l}\text { DCIM-U- } \\
\text { V-II }\end{array}$ & 1 & 1 & $\mathrm{aq}$ & $\begin{array}{c}\text { no } \\
<0614>1116\end{array}$ & $\begin{array}{l}\text { B4.9 } 0925 \\
0923-0928 \\
\end{array}$ \\
\hline $\begin{array}{r}13 \text { Nov. } \\
92 \\
\end{array}$ & $\begin{array}{c}1212: 38 \\
250 \\
\end{array}$ & $\begin{array}{l}1212: 43 \\
200-220 \\
\end{array}$ & $\begin{array}{c}1212: 54 \\
330 \\
\end{array}$ & III-U-N & 1 & 1 & ar & $\begin{array}{c}\text { no patrol } \\
1200-1304 \\
\end{array}$ & no \\
\hline $\begin{array}{r}14 \text { Oct. } \\
90 \\
\end{array}$ & $\begin{array}{l}0903: 04 \\
170-200 \\
\end{array}$ & $\begin{array}{l}\text { 0903:08 } \\
\text { 110-130 } \\
\end{array}$ & $\begin{array}{l}0903: 13 \\
170-200 \\
\end{array}$ & $\mathrm{U}-\mathrm{N}, \mathrm{I}$ & 1 & 1 & (ic) & $\begin{array}{c}\text { no } \\
<0701>1009\end{array}$ & $\begin{array}{l}\text { C1.1 } 0858 \\
0854-0910 \\
\end{array}$ \\
\hline $\begin{array}{r}07 \text { Oct. } \\
90 \\
\end{array}$ & $\begin{array}{l}0850: 48 \\
170-200 \\
\end{array}$ & $\begin{array}{l}0850: 52 \\
110-120 \\
\end{array}$ & $\begin{array}{l}0850: 57 \\
170-200 \\
\end{array}$ & III-U & 1 & 1 & (ic) & $\begin{array}{c}\text { SN N10 E82 } \\
0849-0901 \\
\end{array}$ & $\begin{array}{l}\text { C1.8 } 0851 \\
0847-0855 \\
\end{array}$ \\
\hline $\begin{array}{r}15 \text { Aug. } \\
90 \\
\end{array}$ & $\begin{array}{l}1313: 32 \\
170-240 \\
\end{array}$ & $\begin{array}{c}1313: 36-41 \\
100-130 \\
\end{array}$ & $\begin{array}{c}1313: 50 \\
150 \\
\end{array}$ & U-III & 1 & 1 & (ic) & $\begin{array}{c}\text { no } \\
<1116>1452 \\
\end{array}$ & no \\
\hline $\begin{array}{r}09 \text { Aug. } \\
90 \\
09 \text { Aug. } \\
90 \\
09 \text { Aug. } \\
90 \\
09 \text { Aug. } \\
90 \\
09 \text { Aug. } \\
90\end{array}$ & $\begin{array}{l}0953: 37 \\
170-200 \\
1120: 47 \\
170-200 \\
1312: 29 \\
170-200 \\
1319: 24 \\
170-200 \\
1332: 03 \\
170-200\end{array}$ & $\begin{array}{c}0953: 40 \\
125-140 \\
1120: 50 \\
110-130 \\
1312: 31 \\
(130) \\
1319: 27 \\
110-140 \\
1332: 05 \\
110-140\end{array}$ & $\begin{array}{c}0953: 42 \\
170-200 \\
1120: 55 \\
170-200 \\
1312: 33 \\
? \\
1319: 31 \\
170 \\
1332: 09 \\
170-200\end{array}$ & $\begin{array}{c}\mathrm{U} \\
\text { III-U }\end{array}$ & 1 & $\begin{array}{l}1 \\
2\end{array}$ & $\begin{array}{l}\text { (ic) } \\
\text { (ic) } \\
\text { (ic) } \\
\text { (ic) } \\
\text { (ic) }\end{array}$ & $\begin{array}{c}\text { SF N14 E59 } \\
0945-1017 \\
\text { SF N16 E58 } \\
1113-1120 \\
\text { no } \\
<1120>1315 \\
1 F \text { N13 E60 } \\
1315-1430\end{array}$ & $\begin{array}{c}\text { no } \\
\text { B8.4 } 1318 \\
\begin{array}{c}1317-1319 \\
\text { no }\end{array}\end{array}$ \\
\hline
\end{tabular}

Times and frequencies in Cols. 2-4 refer to the studied type U burst, only. The times are not always referring to the same spectral feature of the burst pattern. The information of this table is not suited to compare burst durations exactly.

H - apparent harmonic emission; F - apparent fundamental emission (cf. Sect. 2.6).

Type - spectral characteristics of the burst group involving the studied type U burst.

Up - number of sources during the ascending U branch (source A). Down - number of sources during the descending U branch (source B + renewed brightening at $\mathrm{A})$.

Loop - type of coronal loop structure: ar - active region loop; aq - active region - quiet region interconnection; ic - interconnecting loop. $\mathrm{H}_{\alpha}$ - Importance and location (first line), start/end of flare from SGD Comprehensive reports; "no": no flare reported during the U burst, end of preceding and start of following reported flare.

SXR - GOES class and peak time (first line), start, end time of the period around the U burst during which the derivative of the $1-8 \AA$ flux is positive. The symbol " $<$ " in the GOES class means that the total flux is given, but the relevant X-ray brightening appears as a minor peak on top of emission from an ongoing flare (estimated accuracy $\pm 0.5 \mathrm{~min}$ ).

(..) - ambiguous information. 


\subsection{Basic pattern of type $U$ burst sources}

Most of the presented observations are consistent with type $\mathrm{U}$ bursts being emitted by electron beams which are guided along large-scale $\left(\sim 1 R_{\odot}\right)$ closed magnetic loop structures as suggested by Labrum \& Stewart (1970):

- At a given frequency the rising and the descending branch are emitted in spatially separated sources (termed A and B in the following, respectively).

- In cases where a single type U burst is imaged at more than one frequency (rare occasion with the standard $\mathrm{NRH}$ frequency setting), the source height increases with decreasing frequency.

- Those type U bursts for which reliable measurements of the large scale photospheric magnetic field are available have their rising and descending branch sources on opposite sides of the magnetic neutral line.

- On several occasions, but not always, the loop structure inferred from the radio observations can be localized above hot and dense coronal plasma structures present in Yohkoh SXT images. These plasma loops connect opposite polarities of one or two active regions or interconnect one active region with an unspecified site of the apparently quiescent atmosphere, respectively (cf. Table 1$)$.

- In the majority of cases where reliable measurements can be made the ascending type $\mathrm{U}$ branch extends to higher frequencies (9 out of 15), has higher flux density $(14 / 23)$ and smaller source size $(12 / 19)$ than the descending branch. However, examples for the opposite situation are found: higher frequency extent of the descending branch (6/15), higher flux density $(9 / 23)$, smaller size $(6 / 19)$. In terms of median values the ascending branch has about 0.9 times the size of the descending branch and 1.4 times its flux density.

- The degree of circular polarization is generally low $(<10 \%)$ and difficult to measure. No systematic behaviour is recognized. The only case where the ascending and descending U-branch have opposite senses of circular polarization is a disk event (like Benz et al. 1979), but other disk events do not show this behaviour.

- The few examples of type U(N) bursts (see Table 1 and e.g. Figs. 17, 19) confirm the basic source geometry and the idea that the electron beam is mirrored in the focussing magnetic field of the descending leg of the plasma loop remote from the beam injection site (Hillaris et al. 1988). The rising spectral lane following the $\mathrm{U}$ burst comes from nearly the same source B as the former descending $\mathrm{U}$ branch. Nevertheless, some details of the spectral data cannot be straightforwardly explained by magnetic mirroring (see e.g. Sect. B.7).

The most clear-cut examples of textbook type U behaviour are:
- the association of two distinct radio sources A and B with active region plasma loops crossing the magnetic inversion line: Fig. 19;

- the alignment along a loop structure of type U burst sources imaged at several frequencies: Fig. 9;

- the only and exceptional case of a type U burst with significant circular polarization of different sign at sites A and B: Fig. 13.

Table 2. Spectral properties of type U bursts

\begin{tabular}{|r|c|r|c|c|c|}
\hline Date & $\Delta f / f_{\mathrm{c}}$ & $f_{\mathrm{s}}-f_{\mathrm{e}}$ & \multicolumn{4}{|c|}{ Relative flux density } \\
& & {$[\mathrm{MHz}]$} & $S_{\mathrm{B}} / S_{\mathrm{A}}$ & $S_{\mathrm{A}^{\prime}} / S_{\mathrm{A}}$ & $S_{\mathrm{N}} / S_{\mathrm{A}}$ \\
\hline 04 Nov. 94 & 0.7 & 65 & 0.6 & 1.1 & - \\
\hline 25 Oct. 94 1007 & 0.3 & -40 & 1.5 & - & - \\
\hline 1144 & 0.5 & -10 & 0.4 & - & - \\
\hline 1231 & 0.9 & -50 & 0.4 & - & - \\
\hline 1331 & $?$ & -110 & 1.5 & - & - \\
\hline 28 Jan. 94 1124 & 0.3 & 40 & 1.2 & - & - \\
\hline 1125 & 0.3 & 60 & - & - & - \\
\hline 1129 & $0.3(\mathrm{H})$ & 30 & 0.9 & - & - \\
\hline 1129 & $0.8(\mathrm{~F})$ & -115 & $\sim 4$ & - & - \\
\hline 25 Jan. 94 & 0.7 & 95 & 0.4 & 0.27 & - \\
\hline 03 Jun. 93 & 0.3 & $(0)$ & 2.9 & - & - \\
\hline 14 May 93 & 0.3 & 40 & $<1$ & - & - \\
\hline 23 Feb. 93 1134 & 0.7 & 80 & 0.9 & 0.48 & - \\
\hline 1138 & 0.7 & 50 & 0.5 & 0.08 & 0.37 \\
\hline 15 Jan. 93 & $(0.8)$ & $(0)$ & 8 & - & - \\
\hline 13 Nov. 92 & 0.4 & -80 & 0.2 & - & 0.05 \\
\hline 14 Oct. 90 & 0.4 & $(0)$ & 1.4 & - & - \\
\hline 07 Oct. 90 & 0.5 & $(0)$ & 1.1 & - & - \\
\hline 15 Aug. 90 & 0.6 & 55 & 0.1 & 0.04 & - \\
\hline 09 Aug. 90 0954 & 0.3 & $(0)$ & $<0.1$ & - & - \\
\hline 1121 & 0.4 & $(0)$ & 0.2 & 0.39 & - \\
\hline 1312 & 0.3 & $?$ & 12 & - & - \\
\hline 1319 & 0.4 & $\geq 0$ & 0.1 & 0.07 & - \\
\hline 1332 & 0.4 & $(0)$ & 0.5 & 0.44 & - \\
\hline & & & & & \\
\hline
\end{tabular}

Columns 2, 3: starting $\left(f_{\mathrm{s}}\right)$, end $\left(f_{\mathrm{e}}\right)$, centre frequency $\left(f_{\mathrm{c}}\right)$, bandwidth $(\Delta f)$ of the $\mathrm{U}$ bursts.

Columns 4-6: flux densities of the sources B (descending branch), $A^{\prime}$ (renewed brightening of the rising branch) and $\mathrm{N}$ (second rising branch of the type $\mathrm{U}(\mathrm{N})$ burst) in terms of the flux density of the rising branch A. They are derived from Gaussian fits to the NRH observations at the best suited frequency (cf. Table 3).

\subsection{More complex source patterns}

\subsubsection{Type U bursts within type III groups}

During many events additional radio sources appear. In general the type $U$ bursts are observed within groups of type III or type J bursts. Only six of the studied events 
Table 3. Spatial properties of type U burst sources

\begin{tabular}{|c|c|c|c|c|c|c|c|}
\hline $\begin{array}{r}\text { Date } \\
\text { (time; freq.) } \\
\end{array}$ & $f_{\mathrm{to}} / f_{\mathrm{NRH}}$ & $\begin{array}{c}x, y \\
{\left[R_{\odot}\right]}\end{array}$ & $\left.\begin{array}{c}r \\
{\left[R_{\odot}\right]}\end{array}\right]$ & $\begin{array}{r}\text { PA } \\
{[\mathrm{deg}]}\end{array}$ & $\begin{array}{l}d_{\mathrm{AB}} \\
{\left[R_{\odot}\right]}\end{array}$ & [beam] & $\begin{array}{l}\mathrm{ze} \\
{[\operatorname{arcmin}]}\end{array}$ \\
\hline $\begin{array}{r}04 \text { Nov. } 94 \\
(11: 39 ; 164) \\
\end{array}$ & 0.77 & $\begin{array}{l}0.06-0.11 \\
0.32-0.22 \\
\end{array}$ & $\begin{array}{l}0.12 \\
0.34\end{array}$ & $\begin{array}{l}209 \\
235 \\
\end{array}$ & 0.28 & $\begin{array}{l}1.4 \\
1.2 \\
\end{array}$ & $\begin{array}{r}1.6 \\
\sim 1.2 \\
\end{array}$ \\
\hline $\begin{array}{r}25 \text { Oct. } 94 \\
(10: 07 ; 435) \\
\end{array}$ & 0.87 & $\begin{array}{l}0.27-0.25 \\
0.30-0.27 \\
\end{array}$ & $\begin{array}{l}0.34 \\
0.40 \\
\end{array}$ & $\begin{array}{l}227 \\
228 \\
\end{array}$ & 0.04 & $\begin{array}{l}1.6 \\
1.9 \\
\end{array}$ & $\begin{array}{l}1.9 \\
2.3 \\
\end{array}$ \\
\hline$(11: 44 ; 164)$ & 0.85 & $\begin{array}{c}0.58-0.17 \\
0.670 .05 \\
\end{array}$ & $\begin{array}{l}0.60 \\
0.67\end{array}$ & $\begin{array}{l}254 \\
274 \\
\end{array}$ & 0.24 & $\begin{array}{l}2.2 \\
3.5 \\
\end{array}$ & $\begin{array}{l}2.7 \\
4.2 \\
\end{array}$ \\
\hline$(12: 31 ; 236)$ & 0.55 & $\begin{array}{c}0.38-0.10 \\
0.480 .17 \\
\end{array}$ & $\begin{array}{l}0.39 \\
0.51 \\
\end{array}$ & $\begin{array}{l}255 \\
290 \\
\end{array}$ & 0.29 & $\begin{array}{l}2.1 \\
4.3 \\
\end{array}$ & $\begin{array}{l}1.9 \\
3.9 \\
\end{array}$ \\
\hline$(13: 31 ; 236)$ & $(0.59)$ & $\begin{array}{c}0.43-0.10 \\
0.510 .05 \\
\end{array}$ & $\begin{array}{l}0.44 \\
0.51 \\
\end{array}$ & $\begin{array}{l}257 \\
276 \\
\end{array}$ & 0.17 & $\begin{array}{l}3.1 \\
3.3 \\
\end{array}$ & $\begin{array}{l}3.8 \\
4.2 \\
\end{array}$ \\
\hline $\begin{array}{r}28 \text { Jan. } 94 \\
(11: 24 ; 236) \\
\end{array}$ & 0.93 & $\begin{array}{ll}1.11 & 0.57 \\
1.07 & 0.60 \\
\end{array}$ & $\begin{array}{l}1.25 \\
1.23 \\
\end{array}$ & $\begin{array}{l}297 \\
299 \\
\end{array}$ & 0.05 & $\begin{array}{l}2.1 \\
2.5 \\
\end{array}$ & $\begin{array}{l}1.9 \\
2.3 \\
\end{array}$ \\
\hline$(11: 25 ; 327)$ & 0.78 & 1.120 .43 & 1.20 & 291 & - & 1.3 & 1.6 \\
\hline$(11: 29 ; 236)$ & 0.91 & $\begin{array}{ll}1.21 & 0.43 \\
1.09 & 0.65 \\
\end{array}$ & $\begin{array}{l}1.28 \\
1.27 \\
\end{array}$ & $\begin{array}{l}290 \\
301\end{array}$ & 0.25 & - & $\begin{array}{l}- \\
-\end{array}$ \\
\hline$(11: 29 ; 164)$ & 0.76 & $\begin{array}{ll}1.21 & 0.69 \\
1.16 & 0.71\end{array}$ & $\begin{array}{l}1.39 \\
1.36\end{array}$ & $\begin{array}{l}300 \\
301\end{array}$ & 0.05 & - & - \\
\hline $\begin{array}{r}25 \text { Jan. } 94 \\
(10: 53 ; 164) \\
\end{array}$ & 0.79 & $\begin{array}{l}-0.570 .15 \\
-0.460 .18 \\
\end{array}$ & $\begin{array}{l}0.59 \\
0.49 \\
\end{array}$ & $\begin{array}{l}75 \\
69 \\
\end{array}$ & 0.11 & $\begin{array}{l}1.7 \\
1.1 \\
\end{array}$ & $\begin{array}{r}2.6 \\
<1.7 \\
\end{array}$ \\
\hline $\begin{array}{r}\text { 03 Jun. } 93 \\
(12: 06 ; 164) \\
\end{array}$ & 0.79 & $\begin{array}{lll}1.05 & 0.52 \\
0.98 & 0.50 \\
\end{array}$ & $\begin{array}{l}1.17 \\
1.10 \\
\end{array}$ & $\begin{array}{l}296 \\
297 \\
\end{array}$ & 0.07 & $\begin{array}{l}2.6 \\
2.4 \\
\end{array}$ & $\begin{array}{l}3.1 \\
2.9 \\
\end{array}$ \\
\hline $\begin{array}{r}14 \text { May } 93 \\
(09: 22 ; 327) \\
\end{array}$ & 0.95 & $\begin{array}{ll}1.08 & 0.26 \\
0.82 & 0.31 \\
\end{array}$ & $\begin{array}{l}1.11 \\
0.88 \\
\end{array}$ & $\begin{array}{l}284 \\
291 \\
\end{array}$ & 0.26 & $\begin{array}{l}1.5 \\
1.2 \\
\end{array}$ & $\begin{array}{r}2.1 \\
\sim 1.6 \\
\end{array}$ \\
\hline $\begin{array}{r}23 \text { Feb. } 93 \\
(11: 34 ; 236) \\
\end{array}$ & 0.8 & $\begin{array}{r}-0.750 .06 \\
-0.630 .03 \\
\end{array}$ & $\begin{array}{l}0.75 \\
0.63 \\
\end{array}$ & $\begin{array}{l}85 \\
87 \\
\end{array}$ & 0.12 & $\begin{array}{l}2.8 \\
3.7 \\
\end{array}$ & $\begin{array}{l}2.4 \\
3.1 \\
\end{array}$ \\
\hline$(11: 38 ; 236)$ & 0.8 & $\begin{array}{c}-0.760 .03 \\
-0.55-0.04 \\
\end{array}$ & $\begin{array}{l}0.76 \\
0.55 \\
\end{array}$ & $\begin{array}{l}88 \\
94 \\
\end{array}$ & 0.22 & $\begin{array}{l}2.0 \\
3.2 \\
\end{array}$ & $\begin{array}{l}1.7 \\
2.7 \\
\end{array}$ \\
\hline $\begin{array}{r}15 \text { Jan. } 93 \\
(09: 25 ; 164) \\
\end{array}$ & 0.67 & $\begin{array}{l}1.30-0.43 \\
0.81-0.97 \\
0.64-0.89 \\
\end{array}$ & $\begin{array}{l}1.37 \\
1.26 \\
1.10 \\
\end{array}$ & $\begin{array}{l}252 \\
220 \\
216 \\
\end{array}$ & $\begin{array}{l}0.80 \\
0.19\end{array}$ & $\begin{array}{r}>3.2 \\
2.8 \\
\end{array}$ & $\begin{array}{l}(11) \\
(10)\end{array}$ \\
\hline $\begin{array}{r}13 \text { Nov. } 92 \\
(12: 13 ; 236) \\
\end{array}$ & 0.9 & $\begin{array}{l}0.55-0.70 \\
0.75-0.52 \\
\end{array}$ & $\begin{array}{l}0.89 \\
0.91 \\
\end{array}$ & $\begin{array}{l}218 \\
235\end{array}$ & 0.27 & $\begin{array}{l}3.5 \\
5.2 \\
\end{array}$ & $\begin{array}{l}2.5 \\
3.7\end{array}$ \\
\hline $\begin{array}{r}14 \text { Oct. } 90 \\
(09: 03 ; 164) \\
\end{array}$ & 0.7 & $\begin{array}{l}-1.33-0.77 \\
-1.61-0.55 \\
\end{array}$ & $\begin{array}{l}1.54 \\
1.70 \\
\end{array}$ & $\begin{array}{l}120 \\
109 \\
\end{array}$ & 0.36 & $\begin{array}{l}2.5 \\
1.5 \\
\end{array}$ & $\begin{array}{l}5.6 \\
3.2 \\
\end{array}$ \\
\hline $\begin{array}{r}07 \text { Oct. } 90 \\
(08: 51 ; 164) \\
\end{array}$ & 0.7 & $\begin{array}{ll}-1.42 & 0.39 \\
-0.98 & 0.42 \\
\end{array}$ & $\begin{array}{l}1.47 \\
1.07 \\
\end{array}$ & $\begin{array}{l}75 \\
67 \\
\end{array}$ & 0.44 & $\begin{array}{l}1.5 \\
1.2 \\
\end{array}$ & $\begin{array}{r}3.4 \\
\sim 1.9 \\
\end{array}$ \\
\hline $\begin{array}{r}15 \text { Aug. } 90 \\
(13: 14 ; 164) \\
\end{array}$ & 0.7 & $\begin{array}{ll}-1.19 & 0.08 \\
-1.46 & 0.09 \\
\end{array}$ & $\begin{array}{l}1.19 \\
1.46 \\
\end{array}$ & $\begin{array}{l}86 \\
86 \\
\end{array}$ & 0.27 & $\begin{array}{l}2.0 \\
2.3 \\
\end{array}$ & $\begin{array}{l}2.5 \\
3.0 \\
\end{array}$ \\
\hline $\begin{array}{r}09 \text { Aug. } 90 \\
(09: 54 ; 164) \\
\end{array}$ & & $\begin{array}{ll}-1.39 & 0.22 \\
-0.97 & 0.56 \\
\end{array}$ & $\begin{array}{l}1.41 \\
1.12 \\
\end{array}$ & $\begin{array}{l}81 \\
60 \\
\end{array}$ & 0.54 & $\begin{array}{l}1.8 \\
2.0\end{array}$ & $\begin{array}{l}2.2 \\
2.4 \\
\end{array}$ \\
\hline$(11: 21 ; 164)$ & 0.7 & -1.380 .30 & 1.41 & 78 & - & 1.9 & 2.3 \\
\hline$(13: 12 ; 164)$ & & $\begin{array}{ll}-1.41 & 0.32 \\
-0.89 & 0.63 \\
\end{array}$ & $\begin{array}{l}1.45 \\
1.09 \\
\end{array}$ & $\begin{array}{l}77 \\
55 \\
\end{array}$ & 0.60 & $\begin{array}{l}2.5 \\
2.6 \\
\end{array}$ & $\begin{array}{l}3.0 \\
3.1 \\
\end{array}$ \\
\hline$(13: 19 ; 164)$ & 0.8 & $\begin{array}{lll}-1.40 & 0.36 \\
-0.91 & 0.67 \\
\end{array}$ & $\begin{array}{l}1.45 \\
1.13 \\
\end{array}$ & $\begin{array}{l}76 \\
54 \\
\end{array}$ & 0.58 & $\begin{array}{l}1.9 \\
2.4 \\
\end{array}$ & $\begin{array}{l}2.3 \\
2.9 \\
\end{array}$ \\
\hline$(13: 32 ; 164)$ & 0.8 & $\begin{array}{ll}-1.38 & 0.32 \\
-0.90 & 0.61 \\
\end{array}$ & $\begin{array}{l}1.42 \\
1.09\end{array}$ & $\begin{array}{l}77 \\
56 \\
\end{array}$ & 0.56 & $\begin{array}{l}1.7 \\
2.1 \\
\end{array}$ & $\begin{array}{l}2.0 \\
2.5 \\
\end{array}$ \\
\hline
\end{tabular}

For each event the first line of Cols. 2-5, 7, 8 refers to the source of the rising branch (A), the second line to the descending branch (B). $x, y$ are the projected positions on the disk with respect to disk centre, referring respectively to the solar east-west and south-north direction (negative values mean east and south, respectively).

$r$ is the projected distance from the centre of the disk, PA the position angle (counted from north over east to south etc.).

$d_{\mathrm{AB}}$ is the projected distance between $\mathrm{UA}$ and $\mathrm{UB}$.

The size is the half-width of the Gaussian fit to the east-west scans of the NRH, corrected for beam broadening if the undeconvolved source width is smaller than 1.5 times the theoretical beam size. It is given as a multiple of the theoretical antenna beam (Col. 7) and in arcmin (Col. 8). 
are not accompanied by type III bursts between $10 \mathrm{~s}$ before and $10 \mathrm{~s}$ after the U burst. Often (e.g. Figs. 16, 17 and 19) the sources of type III bursts and of the type U bursts (A) are spatially separated even if the bursts occurred in time coincidence (cf. also Labrum \& Stewart 1970). In those cases where type III bursts and U bursts occurred within a few seconds, the type III sources were usually (11 out of 12 cases) closer to the ascending branch than to the descending branch of the $U$ burst: the average distance in the plane of the sky to the ascending $U$ sources was found to be $(0.4 \pm 0.25) d_{\mathrm{AB}}$, while the distance to the descending branch source was $(1.2 \pm 0.37)$ $d_{\mathrm{AB}}$, where $d_{\mathrm{AB}}$ is the distance between the two $\mathrm{U}$ burst sources. Other events (e.g. Figs. 21, 22 and 24) are so complex that the association of spectral and spatial features must be inferred by analogy with the clear-cut cases. In some of the spectral records significantly different starting frequencies of quasi-simultaneous type III bursts and U bursts are seen (Figs. 9, 19).

These facts suggest that electron beams are injected in the low corona into structures of different magnetic connectivity. This extends conclusions inferred from earlier imaging observations of type III bursts (Raoult \& Pick 1980; Lantos et al. 1984; Pick \& van den Oord 1990).

\subsubsection{Repeated brightenings near the ascending branch}

In a number of events ( 8 out of 23) a new burst was observed at the site of the ascending branch (A) slightly before or simultaneously with the brightening of the descending branch at site B. There are cases where this renewed brightening is stronger than the descending $U$ branch. Two events may be used to illustrate this situation: In Fig. 4 the renewed brightening (11:39:24 UT) is identified as a negatively drifting (type III) burst. This shows repetitive production of electron beams. On the other hand in Fig. 24 the renewed brightening at site A appears while only a descending $U$ branch is seen in the dynamic spectrum. The fact that this brightening contributes - at least at $164 \mathrm{MHz}$ - twice the flux density of the simultaneous burst at the remote site (cf. Table 2) conflicts with upward travelling beams from the low corona, and seems to require downward reflection of beams by a scattering process near the loop apex (Karlicky et al. 1996). In the other cases the renewed brightening is too weak to allow for a spectral identification.

\subsection{Evidence for harmonically related type U bursts?}

Figures 1 and 12 (Sect. B.3) show the unique case in our sample where a type U burst seems to occur as a pair of harmonically related emission features. The low-frequency lane consists of an ascending branch below $170 \mathrm{MHz}$, followed by a diffuse descending branch extending up to $300 \mathrm{MHz}$. The high-frequency lane $(220-280 \mathrm{MHz})$ is much fainter but gives clear evidence for ascending and
Fig. 1. The event 28 January 1994 11:29 UT. The radio source centroids of the U burst are superposed as crosses onto an enlargement of a Yohkoh SXT full disk image (see Fig. 12). A - the ascending branch; B - the descending branch. F - "fundamental mode" emission (at $164 \mathrm{MHz}$ or $236.6 \mathrm{MHz}$ ); $\mathrm{H}$ "harmonic mode" emission (at 236.6 MHz)

Fig. 2. Composite picture of the source positions of five type U bursts observed during 3h 40m on 09 August 1990. The ascending branch is given by the solid crosses, the descending branch by the hatched crosses. The dimension of the crosses gives the half-width of the undeconvolved sources measured with the east-west and the north-south branch of the NRH 
Fig. 3. 25 October 1994: Position and diameter of the type U burst sources at $435 \mathrm{MHz}$ are superposed on a partial frame Yohkoh SXT image (see text). Lower right: inserted full disk SXT image showing the pre-eruptive active region and the partial frame position at 10:07:39 UT. Compare also Fig. 6

descending branches. The top frequency ratio of both lanes is $1.66 \pm 0.07$, i.e. nearer to a $3: 2$ than to the $2: 1$ ratio. There is no spectral indication of emission at frequencies below $100 \mathrm{MHz}$ which could be identified as the "true fundamental" of this event. Therefore we refer to the low frequency branch as fundamental (F) and to the high frequency branch as harmonic $(\mathrm{H})$ in the following text and tables, but these are to be understood as purely descriptive terms.

At $236 \mathrm{MHz}$ the sources of the ascending and descending $\mathrm{H}$ branch and the descending $\mathrm{F}$ branch occupy three different positions which project upon an arcade of loops seen by Yohkoh-SXT (bottom part of Fig. 12). Figure 1 gives an enlargement of the corresponding part of the SXT image together with the centroid positions of the radio sources. The arcade extends northward behind the limb and is visible in the SXT images already several days before. It is dominated by a very bright loop in the foreground. The two highest type $\mathrm{U}$ sources correspond with the spectral $\mathrm{F}$ lanes at $164 \mathrm{MHz}\left(A_{\mathrm{F}}, B_{\mathrm{F}}\right.$ in Fig. 1). The $236 \mathrm{MHz}$ F source is situated east of the $164 \mathrm{MHz}$ $\mathrm{F}$ sources, at lower height. The $\mathrm{H}$ sources at $236 \mathrm{MHz}\left(A_{\mathrm{H}}\right.$ and $B_{\mathrm{H}}$ ) are situated lower than the $164 \mathrm{MHz} \mathrm{F}$ sources and have a greater mutual distance. To summarize:

1. The sources of the $\mathrm{F}$ and the $\mathrm{H}$ lane align along features with similar geometry as the most prominent X-ray loop, but at larger heights.

2. At $236 \mathrm{MHz}$ the $\mathrm{F}$ source is at lower altitude than the $\mathrm{H}$ sources, whereas it is commonly stated that $\mathrm{F}$ and $\mathrm{H}$ sources at the same frequency are observed at the same height (Dulk 1982, and references therein).
3. The $164 \mathrm{MHz} \mathrm{F}$ emission comes from greater altitude than the nearly simultaneous $236 \mathrm{MHz} \mathrm{H}$ emission, although the inferred electron density would suggest the inverse order. The resolution of this well-known contradiction between the imaging observations and the idea of $\mathrm{F} / \mathrm{H}$ emission is generally searched in electromagnetic wave ducting (Dulk 1982).

4. The projected distance between sources A and B is larger for the $\mathrm{H}$ sources at $236 \mathrm{MHz}$ than for the $\mathrm{F}$ sources at $164 \mathrm{MHz}$, although the $\mathrm{F} / \mathrm{H}$ hypothesis requires that the $236 \mathrm{MHz} \mathrm{H}$ sources are closer to the summit of the beam path than the $164 \mathrm{MHz} F$ source. Ducting (cf. point 3) cannot explain this because when considered separately the $\mathrm{F}$ and $\mathrm{H}$ sources follow the loop geometry outlined by the Yohkoh SXT image (see especially the low projected height of the $B_{\mathrm{F}}$ source !).

Given the contradiction of the observations with the $\mathrm{F} / \mathrm{H}$ emission model, including ducting, we conclude that both lanes in the burst pattern are rather due to a simultaneous injection of different beam ensembles in two nested loops.

\subsection{Repetitive beam injection into stable and evolving structures}

Figure 2 collects in a single graph the $164 \mathrm{MHz}$ positions of five U bursts observed during $3 \mathrm{~h} 40 \mathrm{~min}$ on 09 August 1990 (cf. Figs. 23-27) superposed on the Meudon $\mathrm{H}_{\alpha}$ spectroheliogram. The spectra of the bursts are quite different, but despite their temporal separation the $164 \mathrm{MHz}$ emissions have the same source configuration. A comparison of the time histories of the three events in Figs. 24, 26 and 27 even suggests some degree of similarity in the succession of beam injections during different events: two bursts near the ascending source site A are followed by the brightening of the descending source at B and a simultaneous renewed brightening at $\mathrm{A}$. The observed similarities reveal stable beam injection conditions and a stable magnetic structure which guides the beams.

The flare of 25 October 1994 (Fig. 3; cf. Figs. 69) illustrates a dynamic situation. It is related with evolving coronal structures partly seen by Yohkoh SXT (Manoharan et al. 1996; Aurass et al. 1996). Beginning with the soft X-ray maximum of the flare until several hours later type U bursts repeat at systematically varying sites. Figure 3 shows the $435 \mathrm{MHz}$ radio image of the first burst of the event sequence (see also Fig. 6). It is superposed onto the almost simultaneously recorded Yohkoh SXT partial frame image of a system of sheared flaring loops in AR 7792 (S10 W10) which are under ongoing relaxation. The $\mathrm{U}$ burst is probably associated with newly formed magnetic structures within the active region. Later in the U burst sequence, the site of beam injection changes and the source sites - seen at lower frequencies - are situated in loops bridging another part of the magnetic neutral line far outside the flaring region and having much 
larger span between sites A and B (cf. Figs. 7 to 9). Comparing the bursts of Figs. 8 and 9 at the same frequency $(236 \mathrm{MHz})$, we note again a remarkable repetition of features with a time lag of $1 \mathrm{~h}$ : a starting pair of a type III burst and an ascending type $\mathrm{U}$ branch is followed by the descending type $\mathrm{U}$ branch and a renewed ascending branch.

The two U burst sequences illustrate that the emission may occur in stable as well as in highly dynamic coronal structures. In both cases there is a hint to repetitive sequences of homologous beam injection. Evidence for repetitive U burst emission over several minutes had been given by Aschwanden et al. (1992). Such stable patterns up to several hours appear in contradiction with stochastic or chaotic models of energy release and transport (e.g. Vlahos 1993) and deserve closer investigation.

\subsection{Association of type $U$ bursts with continuum emission}

Figure 18 (Sect. B.8) gives a type U burst with a smooth transition to a type V continuum. Despite this spectral peculiarity, the basic type $U$ pattern of two separate sources $\mathrm{A}$ and $\mathrm{B}$ is present. The descending type $\mathrm{U}$ branch and the continuum are at the same position (B; dashed cross). The source is much bigger than in other events because the emission occurs on top of a complex source which persisted from a preceding burst.

Comparison with the Yohkoh-SXT image suggests that the radio sources are associated with a loop system, and that the site B is in one of the legs. The accuracy of the position measurement is less than in the other events, due to the geometry of the NRH and to residual uncertainties of the ionospheric corrections. Irrespective of this restriction the location of the type $\mathrm{V}$ source at a place different from that of the beam injection is similar to those observed by Dulk et al. (1980). In the present case the observations suggest the continuum emission to come from one side of the loops. Prolonged emission after the descending $\mathrm{U}$ branch is also found in Figs. 20 and 26, although with shorter durations than during the type $\mathrm{V}$ burst. In all three cases the emission comes from the vicinity of the preceding descending $\mathrm{U}$ branch.

\section{Concluding remarks}

Due to the propagation of the emitter along magnetic field lines, the density dependence of their emission frequency and the velocity dependence of their spectral shape type U bursts provide unique diagnostics of coronal plasma - magnetic field structures and their dynamic behaviour, provided sensitive spectral and imaging observations with subsecond time resolution are available. Depending on their frequency, the bursts trace loops seen in soft $\mathrm{X}$-rays (decimetric U bursts) or at greater altitude (metric bursts), where the only other diagnostic is coronographic observation at the limb.
Acknowledgements. The authors are highly obliged to the $\mathrm{YOHKOH}$ community for its open data policy, and to R.D. Bentley (Mullard Space Science Laboratory, U.K.) and his group from the YOHKOH Data Archive Center for support in software and data transfer. They are grateful for the generous supply of GOES soft X-ray observations by the NASA/GSFC Solar Data Analysis Center. We thank our colleagues running the daily observations at Potsdam - Tremsdorf, Nançay and Meudon. Discussions with M. Karlicky, A. Klassen, G. Mann, P.C.H. Martens, M. Pick, A. Raoult, A. Tlamicha, G. Trottet, A.V. Stepanov, L. van Driel-Gesztely, P. Zlobec and Y. Zlotnik are acknowledged, as well as M. Aschwanden's constructive criticism of the manuscript. We thank H. Detlefs and P. Hackenberg for their help in solving software problems, and D. Scholz and G. Servajean for preparing illustrations. The Nançay Radio Observatory is funded by the French Ministry of Education, the CNRS and the Région Centre. This paper became possible due to travel grants 312/pro-bmft-gg (DAAD) and 94053 (MAE) within the German-French PROCOPE programme.

\section{A. Instruments and data reduction}

The observations were carried out jointly at radio wavelengths (Spectrograph of the Tremsdorf Solar Radio Observatory - OSRA, Nançay Radioheliograph - NRH). The OSRA instrument consists of swept-frequency spectrographs in the ranges $40-90,100-170,200-400$ and $400-800 \mathrm{MHz}$, with a sweep rate of $10 \mathrm{~s}^{-1}$ (Mann et al. 1992). The dynamic spectrograms were recorded on film; since September 1993 digitally recorded spectra have been available. The NRH provides two one-dimensional scans of the corona per $0.25 \mathrm{~s}$ (1990), $1 \mathrm{~s}$ (1991-Jan. 1993) and $0.1 \mathrm{~s}$ (thereafter), respectively. In 1990 its east-west branch was operated at $164 \mathrm{MHz}$, the north-south branch at five frequencies (most often 164, 236.6, 327, 408 and $435 \mathrm{MHz}$ ). Since summer 1991 both branches observed at five frequencies (The Radioheliograph Group 1993).

The Meudon spectroheliograph takes one image of the Sun per day. Images in the centre of the $\mathrm{H}_{\alpha}$ line are used in this paper. Yohkoh soft X-ray images and the necessary software were kindly provided by the Yohkoh community. Details are described e.g. in The Yohkoh Analysis Guide (1994).

\section{A.1. Catalogue of burst observations}

By inspection of the OSRA dynamic spectra we selected a sample of type $U$ bursts with branches extending to at least one of the NRH observing frequencies. Those bursts for which NRH data were available were retained for further study. Tables 1 to 3 summarize the observed properties of these bursts. In Figs. 4 to 27 the events are presented using a unified format as follows (from top to bottom):

1. the dynamic spectrum (digital spectra: after background subtraction); 
2. one contour plot of iso-brightness lines, integrated along the direction perpendicular to the baseline of the $1 \mathrm{D}$ antenna array; scans at one frequency from either the east-west or the north-south array are plotted, depending on which provides the most significant information; the position on the ordinate, termed "channel", is given in units depending on the array parameters and the position of the Sun in the sky;

3. the relative brightness (in linear units) as a function of time in an interval of \pm 0.25 channels centered on the centroid position of the rising (solid line) and descending (broken line) branch source of the type $\mathrm{U}$ burst (occasional dips in one curve at the time of maximum in the other are due to sidelobes; e.g. Fig. 17);

4. the map of the radio source positions (centroid and half-power diameters) superposed upon a reference image in soft X-rays (Yohkoh-SXT full disk composite image, see The Yohkoh Analysis Guide, 1994) or $\mathrm{H}_{\alpha}$ (Meudon spectroheliograph). When possible, the position of the relevant part of the neutral line of the large-scale photospheric magnetic field is plotted as a solid line. The position of this line was derived from the full disk magnetograms (Stanford, NOAA Solar Geophysical Data) by using Snodgrass' formula for the differential rotation of magnetic fields (cf. Zirin 1988, Table 6.3).

In order to give the full spectral and spatial information, we usually had to choose different time scales for the plots of the spectrum and the brightness distribution. The $\mathrm{NRH}$ positions and, whenever possible, the flux densities of the different radio sources given in Tables 2 and 3 have been evaluated by a Gaussian (or Gaussian + straight line) fit to the NRH scans. Whenever a significant polarization signature is observed, it is described in the text. The radio positions are plotted as crosses whose extent gives the halfpower diameter of the Gaussian sources measured with the two antenna arrays; the source of the ascending branch is plotted by a solid cross, that of the descending branch as a dashed cross (same line style as the corresponding brightness curves). Corrections for ionospheric refraction were applied using a static standard model or measurements of the ionospheric electron density (correction routines courtesy C. Mercier). The influence of time-dependent density fluctuations was corrected by using simultaneously observed noise storm source locations for reference. Clearly the uncertainty of radio heliographic positions increases with decreasing elevation of the Sun (i.e. in the winter months and far from local noon which is about 11:50 UT at Nançay). Even under worst conditions this uncertainty was smaller than the indicated source size.

\section{B. Description of individual events}

\section{B.1. 4 November 1994}

A type $U$ burst is observed within a group of type III bursts which start near $300 \mathrm{MHz}$ (Fig. 4). Some of them occur nearly simultaneously with the type U.

The source configuration at $164 \mathrm{MHz}$ shows type III bursts at several sites scattered between channels 0 and 2 . The differential radio spectrum (Fig. 5) provides an unambiguous identification of the sources in the iso-brightness contours: the burst at 11:39:17 UT is the ascending type U branch. The type III burst at 11:39:19 UT comes from slightly west of this ascending branch. It coincides in the spectrum with the ascending type U branch - in the differential radio spectrum it is distinctly visible between the top of the type $U$ burst and the low frequency boundary of the spectrogram. At the time of the descending branch (11:39:23 UT) two sources are seen nearly simultaneously: the spectrum shows another type III burst which occurs at $164 \mathrm{MHz}$ slightly after the descending U branch. This timing allows us to identify the weaker western source in the contour plot (channel 6) as the descending U source. The brighter type III emission comes from the site of the earlier type III bursts. The inversion line of the large-scale photospheric magnetic field separates the sources of type III and ascending type $\mathrm{U}$ emission from the site of the descending type $U$ branch. The sources are north of small-scale soft X-ray structures, but have no direct counterpart in the Yohkoh image, perhaps because of its relatively low sensitivity.

\section{B.2. 25 October 1994}

Several type $\mathrm{U}$ bursts are observed during and up to 4 hours after $\mathrm{H}_{\alpha}$-flare-associated type IV continuum and type III burst groups.

The first U burst is identified at 10:07:47 UT (about 17 min after the hard X-ray flare onset) within one minute of the $\mathrm{H}_{\alpha}$ and soft X-ray maximum (Fig. 6 top after background subtraction, which yields gaps at the frequencies of terrestrial transmitters). Although the spectrogram and the contour plot display a complex structure, the timing shows that the brightest $435 \mathrm{MHz}$ sources during the two branches are the sites of type U emission (Fig. 6 bottom). The sources at both 435 and $410 \mathrm{MHz}$ (not plotted in order to avoid confusion) project to opposite sides of the inversion line of the large-scale photospheric magnetic field. They are emitted in a loop which bridges the inversion line in the east-west direction (see also Sect. 2.7).

The U burst at 11:44:15 UT belongs to a group of fastdrift bursts (type III and U/J events) with turnover frequencies in the range 150 to $110 \mathrm{MHz}$. The interval shown in Fig. 7 comprises, in order of increasing time, a type $\mathrm{J}$ burst, the rising branch of the type U, a type III burst which merges with the descending branch of the type $U$ near $150 \mathrm{MHz}$. The weaker burst in the contour plot at 
11:44:18.6 is the descending branch of the type U. Again the type U burst sources project to opposite sites of the photospheric neutral line, but of a different section than the 10:07:47 event, and with beam injection occurring above a region of opposite magnetic polarity.

The U burst pair at 12:31 UT (Fig. 8) is an example of a frequently occurring association with a type III which continues the $\mathrm{U}$ below its spectral turnover. At $236 \mathrm{MHz}$ the source at the time of the ascending branch can be separated into the two components plotted as solid crosses in the bottom panel of Fig. 8. One of these sources is likely the high-frequency part of the type III burst. The descending branch of the first $\mathrm{U}$ burst is observed both at $236 \mathrm{MHz}$ (large dashed cross) and at $327 \mathrm{MHz}$ (smaller dashed cross). These sources are located north of the photospheric magnetic inversion line, while the ascending branch and the type III bursts are south of it. The second $\mathrm{U}$ burst of the pair has the same source configuration, but its descending branch is much weaker.

At 13:31:45 UT a U burst appears together with type III emission during both its rising and descending branch (Fig. 9). The initial type III burst, which extends to frequencies as high as $340 \mathrm{MHz}$, is followed by a sudden flux density increase below $240 \mathrm{MHz}$. The high-frequency envelope of the subsequent emission until $~ 13: 31: 49.5 \mathrm{UT}$ has the typical type U behaviour (we stress, however, that the classification of the different spectral features is not unambiguous).

The event was observed by the NRH at three frequencies: at $164 \mathrm{MHz}$, a single burst traces emission near the turning point of the Type $\mathrm{U}$, while at 236 and $327 \mathrm{MHz}$ two sources are distinguished: on the one hand the initial type III burst at $327 \mathrm{MHz}$ and the rising $\mathrm{U}$ branch at $236 \mathrm{MHz}$, on the other hand the descending branch of the type U. A weak signature of the initial type III burst is seen at $236 \mathrm{MHz}$ (immediately before 13:31:45 UT, at a position slightly west of the much stronger emission from the rising type $\mathrm{U}$ branch). A further type III burst after the descending branch of the type $\mathrm{U}$ is seen at $236 \mathrm{MHz}$ near 13:31:50 UT, at a position close to (but different from) that of the descending type $U$ burst branch.

The centroid positions at different frequencies are plotted by different symbols (one for each frequency) at the bottom of Fig. 9. The extent of the sources, which is similar to the preceding events, is not shown in order to avoid confusion. The configuration is fully consistent with a simple loop structure bridging the inversion line of the photospheric magnetic field and neighbouring open flux tubes near the site of beam injection: the initial type III burst (south of the inversion line) and the descending branch of the type $\mathrm{U}$ at $327 \mathrm{MHz}$ (both plotted as diamonds) enclose the sources at lower frequencies, i.e. the sources of the two branches of the type $\mathrm{U}$ at $236 \mathrm{MHz}$ (triangles) and the single source at $164 \mathrm{MHz}$ (square), which is emitted near the spectral turnover frequency.

\section{B.3. 28 January 1994}

A sequence of several type III/U bursts was observed in the range 100 and $400 \mathrm{MHz}$ between 11:20 and 11:30 UT. Spatially and spectrally related emission features have been noticed earlier (from $09: 47-09: 49 \mathrm{UT}$ ) and spuriously later between 40 and $170 \mathrm{MHz}$ (in the afternoon hours). We present three type $\mathrm{U}$ bursts out of the mentioned interval (Figs. 10 to 12). Note that the SXT image contains some overexposed pixels in the active region of interest.

The spectrum (Fig. 10) shows an asymmetric type U burst turning near $220 \mathrm{MHz}$. The radio image at $236 \mathrm{MHz}$ shows two closely neighbouring sources as expected when the imaging frequency is near to the turning point (compare e.g. Fig. 15).

Figure 11 gives a comparable type $\mathrm{U}$ burst at higher frequencies (turnover $255 \mathrm{MHz}$ ). The $\mathrm{NRH}$ observes only the rising branch at $327 \mathrm{MHz}$. We present this figure because the high starting frequency provides a better tracing of the link with the underlying active region, both since the plasma level is at a lower height in the corona and because the radio propagation is less disturbed by the ionosphere. So we corroborate the association between radio source positions and soft X-ray features made for the other type U bursts of this day which are only observed at lower frequencies.

The spectrum in Fig. 12 is the unique case we found in our data where a type U burst seems to occur as a pair of harmonically related emission features. The top frequency ratio of $1.66 \pm 0.07$ is nearer to the $3: 2$ than to the $2: 1$ ratio. There is no spectral indication of emission at frequencies below $100 \mathrm{MHz}$ which could be identified as the "true fundamental" of this event. From an analysis of the turnover frequency ratio of a sample of $42 \mathrm{U}$ and J bursts Stewart (1974) derives a range of 1.4 to 2.2 for the frequency ratio between harmonic and fundamental emission (mean value $1.8 \pm 0.14$ ). The present case is within this range. Further, Takakura \& Yousef (1974) give evidence for $3: 2$ ratios of related type $J$ burst features. We refer to the low frequency branch as fundamental and to the high frequency branch as harmonic in the following, but these are to be understood as purely descriptive terms. As expected from the faint spectral signature, at $236 \mathrm{MHz}$ the source of the harmonic burst can be located during peaks of emission only. The source configuration is discussed in detail in Sect. 2.6

\section{B.4. 25 January 1994}

The U burst (Fig. 13) is accompanied by a group of type III bursts starting (together with the ascending U branch) near $280 \mathrm{MHz}$. The type III bursts are weak below $170 \mathrm{MHz}$.

Peculiar features of the U burst are a bright patch of $5 \mathrm{MHz}$ bandwidth and $1.7 \mathrm{~s}$ duration near spectral 
turnover and a broadening of the descending branch above $250 \mathrm{MHz}$. The sources in the bottom of Fig. 13 are, from east to west, the ascending $U$ burst branch at $236 \mathrm{MHz}$, then at $164 \mathrm{MHz}$, and the descending branch at $164 \mathrm{MHz}$. The emission at 10:52:59 UT actually starts at a site near the ascending branch, but one second later a brighter source appears farther west. The spectrogram allows for the interpretation that the early emission (10:52:59-10:53:00 UT) is a type III burst which occurs together with the descending $\mathrm{U}$ branch. This would explain the apparent broadening of the latter above $150 \mathrm{MHz}$. The U burst is followed by a more slowly drifting type III burst (10:53:06 UT) which comes from the vicinity of the ascending $\mathrm{U}$ branch source. The $\mathrm{U}$ burst sources at $164 \mathrm{MHz}$ have opposite polarization: $4 \%$ lefthanded during the ascending branch, $6 \%$ right-handed during the descending branch.

Probably due to the low sensitivity of the Yohkoh SXT images available for this day we find no significant soft $\mathrm{X}$-ray features near the radio source positions. The relative location of the radio sources and the active regions is compatible with an interconnecting structure between the two active regions located near E55 S05 and E17 S10. There is no Stanford magnetogram published in SGD on January 25, but a large quiescent filament (e.g. Boulder $\mathrm{H}_{\alpha}$ images, SGD 594-I) suggests that the descending U branch comes from the opposite side of the inversion line of the photospheric field than the ascending branch and the type III bursts.

\section{B.5. 03 June 1993}

The spectrum in Fig. 14 shows a type U like burst with an emission gap after a strong brightening of the ascending branch immediatly before the turning point. Alternatively, the spectrum can be considered as a succession of a type III (but with an unusual kind of low frequency cut-off in the spectrum) and a reverse drift burst. Both burst branches are equally and about $8 \%$ right-handed polarized at $164 \mathrm{MHz}$.

The ascending and the descending sources are close to each other, despite the fact that the $164 \mathrm{MHz}$ observing frequency is well above the turnover frequency of $130 \mathrm{MHz}$. The observations at $164 \mathrm{MHz}$ reveal a similar source structure and evolution during the two burst elements. Each source is embedded in a halo of faint emission (visible e.g. after the peak of the second burst in the contour plots of Fig. 14) which spreads both north-eastward and south-westward from the site of brightest emission, eventually covering a range of $\sim 1 R_{\odot}$. The sensitivity of the analogue spectral record does not permit to identify the spectral characteristics of the spreading emission.

Comparison with the Yohkoh SXT image shows both sources lie above active regions near the limb. Due to projection effects, we cannot decide if the two spectral branches belong to different loop legs. The spreading of the radio emission during the decay phase of the two bursts at $164 \mathrm{MHz}$ occurs transverse to the soft X-ray loops, such as if streams of electrons were injected into a cone of diverging field lines, i.e. a system of east-west oriented loops with a wide range of inclinations to the radial direction. The spectral gap at the turnover frequency suggests that either the radio emission of the beams is cut off near the loop summit or that both branches are due to distinct episodes of beam injection, with the first one producing only an ascending branch, the second one only a descending branch. Type U-like bursts with missing or very faint ascending branches were indeed observed on other occasions (cf. Fig. 25).

\section{B.6. 14 May 1993}

The type U burst shown in Fig. 15 is a rather isolated, unpolarized burst. The rising branch is also observed at $435 \mathrm{MHz}$ with the NRH. Since the NRH observing frequency $327 \mathrm{MHz}$ is near the turnover frequency, no separate sources are seen, but a gradual transition from the ascending to the descending branch site (the eastward broadening of the contours starting at the time of maximum brightness). The rising branch comes from above the western limb at $327 \mathrm{MHz}$ (the bigger solid cross) and at $435 \mathrm{MHz}$ (the smaller solid cross). The descending branch at $327 \mathrm{MHz}$ is on the disk, on the opposite side of the largescale photospheric inversion line. This and the Yohkoh SXT image point to sources in a complex of loops surrounding two active regions near the limb.

\section{B.7. 23 February 1993}

A sequence of two type U bursts has been observed within a group of type III bursts in the range $170-400 \mathrm{MHz}$ between 11:34 and 11:39 UT (Figs. 16 and 17).

The two type U bursts have similar spectra: both turn over near $200 \mathrm{MHz}$, are brighter near the turning point (up to about 1.25 times the turning frequency), and the spectral signature of the rising branch is sharp, while the descending branch appears more diffuse. The 11:38 UT type U burst is followed by a fast-drift burst with a peculiar spectral structure: at frequencies below the end of the preceding descending $U$ branch the drift is negative (type III-like), while no or even a reverse drift is visible at higher frequencies, almost up to the starting frequency of the ascending $\mathrm{U}$ branch.

Both type U bursts display a similar spatial pattern. Three sites of emission can be distinguished:

- The eastern source (channels -18 to -22 ) emits type III bursts. It is simple at 11:38 UT, but comprises a double source at 11:34 UT.

- The source in the center of the complex (channel -16.3 ) brightens when the rising branch of the type $\mathrm{U}$ is visible in the spectrogram. During both U bursts 
successive weaker brightenings occur where the rising branch was emitted.

- The western source brightens during the descending branch of the type U. During the 11:38 UT burst the source has a double structure (separation about 0.3 times the distance between the sites of the ascending and descending source). It is followed by a renewed brightening mainly in the western part of this double structure, during the second ascending branch. This location allows us to identify the event between 11:38:03 and 11:38:15 UT as a type $\mathrm{U}(\mathrm{N})$ burst. While the source positions are compatible with magnetic mirroring of beam particles in a magnetic bottle (e.g. Hillaris et al. 1988), the time delay between the end of the descending $\mathrm{U}$ branch and the start of the second rising $\mathrm{N}$ branch as well as the possible reverse drift of the latter towards higher frequencies argue against a simple and straightforward explanation in terms of a common exciter of the $\mathrm{U}$ burst and the subsequent type $\mathrm{N}$ branch.

The ascending branch of the $\mathrm{U}$ burst (large solid cross at $236 \mathrm{MHz}$, smaller solid cross at $327 \mathrm{MHz}$ ) comes from a site between ARs NOAA 7434 (near S15 E65) and 7433 ( $\sim \mathrm{N} 15$ E45), while the descending branch as well as the second rising branch are situated on the opposite side of the large-scale neutral line, near the following part of AR 7432 ( $\sim$ S15 E20) where the subflare is reported. The beam injection site is therefore not directly related with the subflare (cf. Table 1).

The comparison of the type $U$ radio source sites with soft X-ray features provides an intriguing result: the radio sources are aligned along a faint soft X-ray structure connecting ARs NOAA 7433 and NOAA 7432. But the source position of the $327 \mathrm{MHz}$ ascending branch, which should be closer to the site of beam injection, rather suggests the south-eastern AR NOAA 7434 as a host for the beam injection site. So do the type III bursts (not shown in the figures) which come from a source east of the rising type $\mathrm{U}$ branch and are further displaced from the soft $\mathrm{X}$-ray feature in the direction to AR 7434 .

\section{B.8. 15 January 1993}

The spectrum in Fig. 18 shows a well-defined U burst with a strong type $\mathrm{V}$ continuum tail after its descending branch (see also Sect. 2.8). In the frequency range 145$170 \mathrm{MHz}$ two type III-like bursts are seen between the rising and the descending branch. The radio emission in the range $100-250 \mathrm{MHz}$ belongs to a complex event ( $\mathrm{d} m$ and $m$ activity earlier, metric type II activity later) related to a flare behind the western limb.

Three radio sources are distinguished at $164 \mathrm{MHz}$. During the ascending branch two sources brighten in close succession (channels 20 and 14). Both were already acting during the preceding activity. Since the two sources make a comparable contribution to the $164 \mathrm{MHz}$ flux density dur- ing the ascending branch, both are plotted as solid crosses upon the Yohkoh-SXT image (Fig. 18, bottom). The descending branch and the continuum occur at the same place (dashed cross) at lower projected altitude than the ascending branch. The big source size is due to the superposition of the new emissions on the broad and complex ongoing emission.

Besides the confusion with the ongoing event, the observing conditions at low elevation in winter are unfavourable to get a clear picture of the source configuration and its association with soft X-ray features. When projected on the sky, the two baselines of the NRH form an angle significantly different from $90^{\circ}$. As a consequence, errors in the one-dimensional positioning yield bigger uncertainties of the heliographic positions than in the other events studied. Residual effects of ionospheric gravity waves add to the uncertainty. As a result, the relative positioning between radio sources and soft X-ray features is subject to an uncertainty of $0.1-0.2 R_{\odot}$. Within this restriction, the type $\mathrm{U}$ sources project upon opposite sides of a large-scale loop system above the south-western limb.

\section{B.9. 13 November 1992}

The spectrum (Fig. 19) shows a strong type III burst followed by a fainter type U burst with lower starting frequency than the type III. The U burst is followed by a faint, short brightening which gives the aspect of a type $\mathrm{U}(\mathrm{N})$ burst. However, the spectral record does not reveal a clear finite drift rate. The brightness distribution shows the position of the initial type III burst until 12:12:42 UT. The subsequent peak is slightly west of the type III position. We identify this site as the source of the ascending branch of the type $U$ burst and attribute the westward displacement to its brightening at a place close to, but different from, the preceding type III burst (projected distance $0.04 R_{\odot}$ ). The descending branch has its source west of the rising branch. The following source (12:12:50 UT) is close to the preceding descending branch. This confirms its identification as a type $\mathrm{U}(\mathrm{N})$ burst (like Fig. 17).

Comparison with the Yohkoh image and the magnetic inversion line reveals that the sources are situated in an active region loop overlying a system of soft X-ray loops. The type $\mathrm{U}$ burst occurred in conjunction with a soft $\mathrm{X}$-ray jet in the trailing part of the active region, underneath the sites of emission during the type III burst and the rising branch of the type $U$ (Aurass et al. 1994).

\section{B.10. 14 October 1990}

The type U burst (Fig. 20) has well-defined ascending and descending branches and a bright and diffuse extension around the turnover point and the beginning descending branch. A faint third branch (possibly $\mathrm{U}(\mathrm{N})$ ) is seen in the 
low frequency part of the spectrum $(110-130 \mathrm{MHz})$ after 9:03:20 UT. At frequencies below the turning point of the type $\mathrm{U}$ the ascending branch is continued as a faint type III burst until less than $40 \mathrm{MHz}$. The narrow-band bursts seen in the spectrogram are associated with a noise storm which is well separated from the U burst at $164 \mathrm{MHz}$.

The $164 \mathrm{MHz}$ emission has two well separated sources. The rising branch comes from a source which appears double in itself. The dominant component lies above the south-eastern limb. After a detailed comparison with other bursts on the same day we conclude that the secondary source is a sidelobe due to noise storm emission at a remote site. The descending branch comes from a distinct simple source north-eastward of the rising branch. After its end a new rise is visible at the same site from 09:03:15 to 09:03:20 UT, which might be the high-frequency part of the above mentioned faint third branch of the burst. In this case the event would be of type $\mathrm{U}(\mathrm{N})$.

The radio sources lie above AR's $6321(\sim \mathrm{S} 25 \mathrm{E} 50)$ and 6322 ( $\sim$ S20 E60) near the south-eastern limb as seen by superposition on the daily image of the Meudon spectroheliograph. The relative position of the two U sources is compatible with radio emission from a large loop structure that interconnects AR 6321 with AR 6322 or with a quiet region in the photosphere.

\section{B.11. 7 October 1990}

The type U burst is observed among a diffuse background of type III bursts (Fig. 21) which start above $170 \mathrm{MHz}$ and partly extend to below $100 \mathrm{MHz}$. The background emission is interrupted e.g. during the $\mathrm{U}$ burst at frequencies below the burst trace. Like in the case of 14 October 1990 the turning region is much brighter than both branches, but there is no type $\mathrm{V}$ like continuum extension.

Two source sites are distinguished during the onset of the burst (channels -18 and -14 ). Both were already acting during the preceding type III emission. The brightness maximum of the eastern source (08:50:49 UT) fits in time with the spectral signature of the ascending $U$ branch. The analogue spectral record does not indicate the emission of the western source site due to its relative weakness. The descending type $U$ burst source is most intense and consists of a close succession of two brightenings at channels -13.75 and -13.5 (08:50:57 UT). All radio sources are unpolarized.

The combined plot of type $\mathrm{U}$ source positions and the spectroheliogram is inconclusive as to the associated loop structure.

\section{B.12. 15 August 1990}

In Fig. 22 a cluster of type $\mathrm{J}$ and $\mathrm{U}$ bursts is shown with turnover frequencies in the range $130 \mathrm{MHz}$ to below $100 \mathrm{MHz}$.
The dominant emission at $164 \mathrm{MHz}$ comes from three successive negatively drifting bursts (13:13:3013:13:42 UT). They are also observed at $236 \mathrm{MHz}$. The first of these bursts turns below $110 \mathrm{MHz}$, the following two merge with type $U$ bursts which turn slightly above $130 \mathrm{MHz}$. The position and size of the brightest burst at $164 \mathrm{MHz}(13: 13: 34 \mathrm{UT})$ is plotted by the solid cross in the bottom panel of Fig. 22 upon the $\mathrm{H}_{\alpha}$ spectroheliogram. The burst at 13:13:40 UT is at the same position, while that at 13:13:37 UT is situated $\sim 0.02 R_{\odot}$ north-east. The sources have asymmetric profiles in the east-west scans. This means that at least two neighbouring sources contribute to each of the bursts or that there is the same kind of spreading as on 03 June 1993. After the fade-out of the negatively drifting bursts at $164 \mathrm{MHz}$, a faint signature of the descending branch of one of them becomes visible (13:13:45 UT) east of the source of the negatively drifting bursts (channel -22 in the iso-brighness representation of the east-west NRH branch; not strong enough for the amplitude curve; dashed cross, bottom of Fig. 22). Its heliographic position is not accurately determined because the NRH data compression procedure does not detect significant changes in the north-south scans between 13:13:42 and 13:13:44.5 UT. Weak burst activity continues afterwards near the site of the previous negatively drifting bursts.

We cannnot decide which of several active regions near and behind the limb are connected by the loop structure hosting the type $\mathrm{U}$ source.

\section{B.13. 09 August 1990}

Within $3 \mathrm{~h} 40 \mathrm{~min}$ five similar burst groups have been identified in the range 100-170 MHz. The spectra in the top of Figs. 23 to 27 show mostly interlaced type III and type U bursts.

While the spectra are quite different, the NRH data reveal a similar source configuration in all cases. The ascending branches and type III bursts come from an eastern source (channel -23 to -21 ). These sources have asymmetrical contours that extend toward the later appearing descending branch source near channel -15 . The descending branch cannot always be identified in the spectral records, but is the dominant feature of the 9:53:30 UT event. A faint brightening is simultaneously observed with the descending site source at the position of the ascending source.

The bursts are emitted in basically the same large-scale coronal loop structure (see also Sect. 2.7). The sources of the negatively drifting bursts are somewhat scattered in position. Most of them are intrinsically complex. The source of the descending branch undergoes an apparent shift away from the source of the rising branch.

Both timing and source geometry suggest that the rising branch source of the type $\mathrm{U}$ bursts is connected with the flaring active region NOAA 6197 . Given the 
north-westward displacement of the descending branch source with respect to the rising branch, the small plage region at $\mathrm{N} 37 \mathrm{E} 40$ visible in the $\mathrm{H}_{\alpha}$ spectroheliogram (bottom of Figs. 23 to 27 ) is a plausible site for a conjugate footpoint of a large-scale magnetic loop.

No significant polarization is observed during the ascending branch of type U bursts. Only in the 13:32 UT event about $10 \%$ left-handed polarization is detected during the descending branch.

\section{References}

Aschwanden M.J., Bastian T.S., Benz A.O., Brosius J.W., 1992, ApJ 391, 380

Aurass H., Klein K.-L., Martens P.C.H., 1994, Solar Phys. 155, 203

Aurass H., Klein K.-L., Martens P.C.H., 1996, in: Bentley R.D., Mariska J.T. (eds.) Proc. Yohkoh Conference on Magnetic Reconnection in Solar Flares, Astr. Soc. Pac. Conf. Ser. 111, 194

Benz A.O., Berney M., Santin P., 1977, A\&A 56, 123

Benz A.O., Urbarz H.W., Zlobec P., 1979, A\&A 79, 216

Bray R.J., Cram L.E., Durrant C.J., Loughhead R.E., 1991, Plasma Loops in the Solar Corona. Cambridge University Press, Cambridge

Caroubalos C., Poquérusse M., Bougeret J.L., Crepel R., 1987, ApJ 319, 503

Dennis B.R., Zarro D.M., 1993, Solar Phys. 146, 177

Dulk G.A., 1982, in: Benz A.O., Zlobec P. (eds.) Proc. 4th CESRA Workshop on Solar Noise Storms. Trieste Observatory Publication, p. 356

Dulk G.A., Suzuki S., Gary D.E., 1980, A\&A 88, 218

Fokker A.D., 1970, Solar Phys. 11, 92

Goldman M.V., Smith, D.F., 1985, in: Sturrock P.A. (ed.) Physics of the Sun. Vol. II. Reidel, Dordrecht, p. 325

Haddock F.T., Takakura T., pers. comm. in Kundu M.R., 1965, Solar Radio Astronomy. Interscience Publishers, New York, p. 311
Hillaris A., Alissandrakis C.E., Vlahos L., 1988, A\&A 195, 301

Karlicky M., Mann G., Aurass H., 1996, A\&A 314, 303

Labrum N.R., Stewart R.T., 1970, PASA 1, 316

Lantos P., Pick M., Kundu M.R., 1984, ApJ 283, L71

Mann G., Aurass H., Voigt W., Paschke J., 1992, in: Coronal Streamers, Coronal Loops and Coronal and Solar Wind Composition, ESA SP-348, p. 129

Manoharan P.K., van Driel-Gesztely L., Pick M., Démoulin P., 1996, ApJ 468, L73

Maxwell A., Swarup G., 1958, Nat 181, 36

Pick M., van den Oord G.H.J., 1990, Solar Phys. 130, 83

Pick M., Raoult A., Trottet G., Vilmer N., Strong K., Magalhaes A., 1994, in Enome S., Hirayama T. (eds.) Proc. Kofu Symp. NRO Report 360, Nobeyama Radio Observatory, p. 263

Priest E.R., 1978, Solar Phys. 58, 57

Raoult A., Pick M., 1980, A\&A 87, 63

Raoult A., Vlahos L., 1995, A\&A 296, 844

Sheridan K.V., McLean D.J., Smerd S.F., 1973, Astrophys. Lett. 15, 139

Solar Geophysical Data (SGD), 1990 - 1995, National Oceanic and Atmospheric Administration (ed.), U.S. Dept. of Commerce, Boulder, Colorado, U.S.A.

Stewart R.T., 1974, Solar Phys. 39, 451

Stewart R.T., Vorpahl J., 1977, Solar Phys. 55, 111

Stone R.G., Fainberg J., 1971, Solar Phys. 20, 106

Suzuki S., 1978, Solar Phys. 57, 415

Suzuki S., Dulk G.A., 1985, in: McLean D.J., Labrum N.R. (eds.) Solar Radiophysics. Cambridge University Press, Cambridge, p. 289

The Radioheliograph Group, 1993, Adv. Space Res. 13(9), 411

Takakura T., Yousef S., 1974, Solar Phys. 36, 451

The Yohkoh Analysis Guide, 1994, compiled by M. Morrison Vlahos L., 1993, Adv. Space Res. 13(9), 161

Zirin H., 1988, Astrophysics of the Sun. Cambridge University Press, Cambridge 


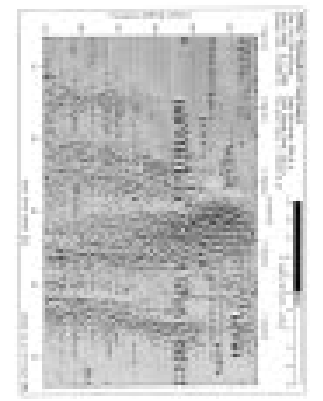

Fig. 4. Spectrum and source configuration during a type U burst. From top to bottom: 1) Dynamic spectrum (linear frequency scale). 2) One-dimensional brightness distribution (integrated in the direction perpendicular to the array). Vertical axis: $0=$ disk centre, total field of view 64 channels. Linear contours, $20 \%-100 \%$ of maximum. 3) Flux density within \pm 0.25 channels around the position of maximum brightness (solid: ascending branch, dashed: descending branch). 4) Yohkoh-SXT image (13:28:29 UT, AlMg filter, $7.5 \mathrm{~s}$ exposure), radio source positions and sizes (cf. Fig. 2) and inversion line of the large-scale photospheric magnetic field (Solar Geophysical Data)

Fig. 5. The time derivative of the 4 November 1994 event spectrum shown in Fig. 4, top, clearly reveals a type III burst crossing the turning point of the type U burst 
Fig. 6. NRH contour levels: linear spacing, $10 \%$ - 100\% of maximum brightness, after subtraction of a pre-event scan. Yohkoh image: 0953:19 UT, filter AlMg, exposure time 30 s composed with earlier less exposed images. Compare also Fig. 3

Fig. 7. NRH (north-south branch) contours: linear, $4.3 \%$ - $100 \%$ of maximum brightness 
Fig. 8. NRH (north-south) contours: NRH linear, $10 \%-100 \%$ of maximum brightness

Fig. 9. NRH (north-south) contours: linear, $10 \%-100 \%$ of maximum brightness. Bottom: centroid positions of the radio sources at $164 \mathrm{MHz}$ (square), $236 \mathrm{MHz}$ (triangles), $327 \mathrm{MHz}$ (diamonds; southern source = initial type III burst, northern source $=$ descending $\mathrm{U}$ branch) 
Fig. 10. NRH contours: linear, $20 \%$ - 100\% of maximum brightness. Yohkoh: 10:53:54 UT, AlMg, 5.3 s exposure time

Fig. 11. NRH contours: linear, $4.3 \%-100 \%$ of maximum brightness. Yohkoh: cf. Fig. 10 
Fig. 12. NRH (north-south) contours: linear, $20 \%-100 \%$ of peak brightness, after subtraction of a pre-event scan). Yohkoh: cf. Fig. 10

Fig. 13. NRH contours: linear, $10 \%$ - 100\% of peak brightness. Yohkoh: 1103:51 UT, AlMg, 0.7 s exposure 
Fig. 14. NRH contours: linear, $4.3 \%$ - 100\% of the peak value. Yohkoh: 1257:49 UT, AlMg, composed of a 30 s exposed picture with earlier ones of shorter exposure time

Fig. 15. NRH contours: logarithmic, $5 \%$ - 100\% of maximum brightness. Yohkoh: 0923:53 UT, AlMg, 0.7 s exposure 
Fig. 16. NRH contours: linear, $4.3 \%$ - 100\% of maximum brightness. Yohkoh: 1254:12 UT, AlMg, 30 s exposure, composed with earlier less sensitive images

Fig. 17. See caption of Fig. 16 
Fig. 18. NRH contours: logarithmic, 5\% - 100\% of maximum brightness. Yohkoh: 0701:41 UT, AlMg, composed of a $30 \mathrm{~s}$ exposure and earlier less sensitive images

Fig. 19. NRH contours: logarithmic, $1 \%$ - 100\% of maximum brightness. Yohkoh: 1213:12 UT, Al.1, 2.7 s exposure. The image reveals a soft X-ray jet starting one minute before electron beam injection in the ascending loop leg 
Fig. 20. NRH contours: linear, $4.3 \%-100 \%$ of maximum brightness. Meudon $\mathrm{H}_{\alpha}$ spectroheliogram: 0858 UT

Fig. 21. NRH contours: linear, $4.3 \%-100 \%$ of maximum brightness. $\mathrm{H}_{\alpha}$ : $0902 \mathrm{UT}$ 
Fig. 22. NRH contours: logarithmic, $1 \%-100 \%$ of maximum brightness. $\mathrm{H}_{\alpha}$ : $0611 \mathrm{UT}$

Fig. 23. NRH contours: linear, $4.3 \%-100 \%$ of maximum brightness. $\mathrm{H}_{\alpha}$ : $0705 \mathrm{UT}$ 
Fig. 24. See caption Fig. 23. The two simultaneous sources during the descending branch are confused in the scans with the north-south branch of the NRH. Only the position of the ascending branch is plotted in the bottom panel

Fig. 25. See caption Fig. 23 
Fig. 26. See caption Fig. 23

Fig. 27. See caption Fig. 23 